\title{
Del enfoque por tareas a la perspectiva co-accional*
}

\author{
Christian Puren** \\ Université de Saint-Étienne, France \\ Centres d'Études en Didactique Comparée des Langues Et des Cultures, CEDICLEC
}

\section{RESUMEN}

Se presenta una comparación entre "enfoque por tareas", como lo desarrollaron los didactas anglo-sajones dentro del enfoque comunicativo, y la "perspectiva accional" del reciente Marco europeo común de referencia del Consejo de Europa (2001). Interesa distinguir entre tareas (de aprendizaje) y acciones (sociales, fuera del aula), y mantener el concepto de "tarea" en el nivel de abstracción -transmetodológico-, pues permite distinguir entre diferentes "orientaciones" posibles de las tareas. Por ello, es necesario mantener el concepto de "perspectiva accional" del Consejo de Europa, el de "representación de la relación entre tarea y acción", ya que las metodologías tuvieron en el pasado su propia "perspectiva accional". Finalmente, se designa dicha perspectiva con el nombre de "perspectiva co-accional": su implicación más original consiste en otorgar un privilegio a las tareas encaminadas a la preparación en clase de acciones colectivas con finalidad social.

\section{ABSTRACT}

This article presents a comparison between a 'task approach', as it was developed by the British didactics within the communicative approach, and the 'actional perspective' proposed by the Common European Framework (2001). It is interesting to distinguish between tasks (learning) and actions (social ones, out of the context of a classroom), and on the other to keep the concept of 'task' in its abstract meaning-transmethodologic- so that it allows us to distinguish between the different possible 'orientations' within the tasks. Here lies the reason of the importance of keeping the concept of 'actional perspective' coined by the European Council, as 'it represents the relationship between task and action'. Because the methodologies hold in the past their own 'actional perspective'. Finally this perspective is known as 'co-actional perspective': its main implication consists in fostering the tasks aimed to prepare collective actions in the class with a social purpose.

* El artículo, fue publicado en la revista Porta Linguarum: revista internacional de didáctica de las lenguas extranjeras (ISSN 1697-7467) №. 1, 2004 , págs. 31-36. Se mantiene forma original de citación.

** Christian Puren ha sido profesor de francés y español en varias universidades de Francia y en otros países. Es presidente de Honor de la APLV (Association française des Professeurs de Langues Vivantes) y del GERES (Groupe d'Etude et de Recherche en Espagnol de Spécialité); además es profesor emérito de la Universidad Jean Monnet de Saint-Étienne (Francia) desde septiembre de 2008, y miembro de los comités científicos de varias revistas francesas y extranjeras en el área de la Didáctica de las Lenguas-culturas. Es autor de Histoire des méthodologies de l’enseignement des langues (1988), La didactique des langues à la croisée des méthodes. Essai sur l’éclectisme (1994), entre otras obras y artículos. 
Es bien conocido desde hace años en la didáctica anglosajona y en España el "enfoque por tareas". Me propongo, en este artículo, mostrar las diferencias que existen entre dicho enfoque y la nueva orientación que se esboza en el último documento del Consejo de Europa sobre enseñanza/aprendizaje de las lenguas -el Marco europeo común de referencia ${ }^{3}$-, orientación que sus autores llamaron "perspectiva accional". Las líneas más interesantes a este respecto son las del capítulo $3^{\circ}$ de la edición provisional de 1996, en el que presentan la nueva perspectiva con estos términos:

Un marco de referencia debe situarse con relación a una representación muy general del uso y del aprendizaje de las lenguas. La perspectiva que privilegiamos aquí es, muy generalmente también, de tipo accional en el sentido en que considera ante todo el usuario y el alumno de una lengua como actores sociales que deben realizar tareas (que no sólo son lingüísticas) en unas circunstancias y un ámbito determinado, dentro de un área particular de acción. Si bien los actos de habla se realizan dentro de actividades lingüísticas, éstas se realizan a su vez dentro de acciones sociales indispensables para darles su pleno significado. (Traducción del autor)

Veremos sucesivamente cómo estas pocas líneas inician una salida del enfoque comunicativo con un paso del concepto de "acto de habla" al de "acción social", pero también cómo dos nuevas distinciones - entre "tarea" (de aprendizaje) y "acción" (social) y entre "perspectiva accional" y "perspectiva co-accional" - permiten completarla, para proponer a los profesores una nueva coherencia de enseñanza/aprendizaje susceptible de enriquecer las herramientas de las que disponen actualmente.

\section{Del"acto de habla" a la"acción social"}

En este párrafo los autores del Marco europeo común efectúan una primera distinción conceptual entre el

3 Marco europeo común de referencia para el aprendizaje y la enseñanza de las lenguas. Estrasburgo: Comité de la educación, Consejo de Europa, edición provisional 1996 [2a ed. provisional 1998, ed. Definitiva 2001. acto definido lingüísticamente (los "actos de habla" o "funciones lingüísticas" tales como saludar, agradecer, pedir, disculparse, etc.) y la acción definida socialmente. Al señalar además que no todas las tareas son lingüísticas, ellos, implícitamente pero muy claramente, toman así sus distancias con relación al enfoque comunicativo dominante en Europa desde la publicación por el mismo Consejo de Europa, en los años 70, de los diferentes "Niveles umbrales" (e.g. The Threshold level, 1972; Un Niveau-seuil, 1975).

Ahora bien, uno de los autores de referencia entre los metodólogos anglosajones en cuanto a "enfoque por tareas", David Nunan, define así el concepto de "tarea":

Task: a piece of classroom work which involves learners in comprehending, manipulating, producing or interacting in the target language while their attention is principally focused on meaning rather than form. (Nunan, 1989,19)

Es evidente que la definición que da D. Nunan de "tarea" es la de "tarea comunicativa" (cf. "interacting", "focused on meaning rather than form"), y que le ha dado al concepto un sentido que corresponde estrictamente a la ortodoxia didáctica vigente en ese momento (la del enfoque comunicativo). Este es un fenómeno constante en la historia de las metodologías: huelga decir que en tiempos de la metodología gramática-traducción, las tareas de referencia eran naturalmente las tareas gramaticales y las tareas de traducción...

Esta reducción permanente de los conceptos didácticos fundamentales dentro del marco delimitado por las coherencias metodológicas sucesivas ${ }^{4}$ se justifica desde un punto de vista interno (se trata así de reforzar la coherencia conceptual de conjunto de cada

4 Se puede usar una metáfora para describir este fenómeno: los conceptos son como esponjas que constantemente van embebiéndose de los elementos de su entorno conceptual... 
metodología), pero tiene sus efectos negativos. En particular, hace muy difícil la comparación intermetodológica, procedimiento básico para la formación didáctica de los docentes porque sólo ella permite después en clase la diversificación de los procedimientos, indispensable para atender a la diversidad de los alumnos y de los entornos de enseñanza/ aprendizaje. Por eso es imprescindible, en contra de las expectativas concretas de muchos profesores y de las orientaciones empíricas de muchos formadores, mantener la definición de los conceptos didácticos en su mayor nivel de abstracción. Una de las pocas leyes "científicas" de nuestra disciplina es en efecto la siguiente: "Tanto más concreto es un concepto, cuanto más restringido es su uso al contexto preciso en el que se elaboró"

Por eso me parece que la mejor definición posible de "tarea", es la más abstracta, que se podría enunciar así: "unidad de sentido dentro del proceso conjunto de aprendizaje y enseñanza”. Para demostrar a contrario la ley enunciada más arriba, propongo a continuación un cuadro en el que presento las diferentes orientaciones que se le puede dar en didáctica de las lenguas a la tarea definida de esta manera tan amplia:

\section{Tipos de orientación de las tareas}

Ejemplo: consignas posibles sobre la instrucción de montaje de un aparato (propuesta en L1 o L2)

Tabla 1.

\begin{tabular}{|l|l|l|}
\hline \multicolumn{1}{|c|}{ Consigna } & Orientación de la tarea & Criterio de Evaluación \\
\hline $\begin{array}{l}\text { 1. Reescriba esta instrucción pasando del voceo al tuteo. } \\
\text { 2. Escoja entre las siguientes diferentes traducciones de } \\
\text { esta instrucción, y explique las razones de su elección }\end{array}$ & lengua & Corrección de la forma \\
\hline $\begin{array}{l}\text { 3. En grupo de dos: un utilizador no entiende ciertos } \\
\text { puntos de su instrucción en lengua extranjera; el otro se } \\
\text { los explica }\end{array}$ & comunicación & gestión del aprendizaje \\
\hline $\begin{array}{l}\text { 4. Usted quiere convencer a otro de que su propia tra- } \\
\text { ducción en L2 de la instrucción es mejor que aquélla de } \\
\text { la que él dispone. }\end{array}$ & resultado & buscado \\
\hline $\begin{array}{l}\text { 5. Traduzca la totalidad de esta instrucción teniendo en } \\
\text { cuenta el estilo de las notas para aparatos de este tipo en la } \\
\text { el país extranjero (ver ej. adjuntos). }\end{array}$ & transmisión del sentido \\
\hline $\begin{array}{l}\text { 6. La sociedad inventora de aparato le pide que le pro- } \\
\text { ponga la correspondiente instrucción de montaje : re- } \\
\text { dáctela, pruébela con algunos utilizadores, luego pro- } \\
\text { ponga una traducción con variantes argumentadas que } \\
\text { permitan a esa sociedad tomar posteriormente sus pro- } \\
\text { pias decisiones sobre el texto final que ella seleccionará. }\end{array}$ & producto & adecuación cultural de \\
\hline
\end{tabular}




\begin{tabular}{|l|l|l|}
\hline \multicolumn{1}{|c|}{ Consigna } & Orientación de la tarea & \multicolumn{1}{|c|}{ Criterio de Evaluación } \\
\hline $\begin{array}{l}\text { 7.Realice sucesivamente las tareas siguientes: } \\
\text { a) Ponga los esquemas siguientes de montaje en su } \\
\text { orden lógico. }\end{array}$ & & realización correcta en \\
$\begin{array}{l}\text { b) Ponga en el orden correspondiente las frases siguien- } \\
\text { tes de la instrucción. }\end{array}$ & procedimiento & $\begin{array}{l}\text { su debido orden de } \\
\text { cada una de las tareas } \\
\text { c) Añada los conectores cronológicos adecuados escogi- } \\
\text { dos en la lista adjunta }\end{array}$ \\
\hline
\end{tabular}

na sola de esas siete tareas, la número 3, es "comunicativa" en el sentido que se le da generalmente a la "interacción" en el enfoque comunicativo - el de intercambio de informaciones entre dos interlocutores- ${ }^{5}$ pero yo no veo por qué razón un profesor prescindiría a priori de cualquiera de las demás. El problema no es saber cuál es la mejor in se et per se, sino escoger cuál va a ser la más adecuada al entorno del momento (a qué alumnos, qué objetivos, qué soporte, etc.) y la más coherente con el dispositivo elaborado en función de este entorno. En otros términos, tenemos que pasar de ahora en adelante, en didáctica de las lenguas, de un paradigma de la optimización (en el que se pretende encontrar los mejores procedimientos universales y permanentes) a un paradigma de la adecuación (en el que se pretende buscar en cada situación y en cada momento el procedimiento más eficaz entre todos los disponibles). ${ }^{6}$

\section{Tarea de aprendizaje y acción social}

En el párrafo citado, los autores del Marco europeo común establecen otra distinción entre usuario/uso, por una parte, y alumno/aprendizaje, por otra par-

5 La número 5 también obligaría a secuencias de intercambio de informaciones, de modo que los metodólogos comunicativistas probablemente estarían dispuestos a apropiársela...

6 En cierta tradición anglosajona, resulta muy paradójico, si lo pensamos bien, el hecho de que la orientación empírica sea muy fuerte, pero al mismo tiempo que ella esté estrictamente encuadrada dentro de la ortodoxia didáctica vigente. te, distinción que me parece interesante utilizar para oponer "tarea" y "acción": propongo llamar "tareas" lo que hacen los alumnos en clase durante su proceso de aprendizaje, y “acción” lo que tienen/ tendrán que hacer con la lengua extranjera en la sociedad durante su aprendizaje y/o al final. Los autores del Marco europeo común no hacen tal distinción (cf. los “actores sociales que deben realizar tareas..."), sin duda porque siguen estando influidos por el marco conceptual del enfoque comunicativo, en el que la actividad de referencia - la simulación - se caracteriza precisamente por una homología perfecta entre tarea de aprendizaje y acción en la sociedad, fingiendo los alumnos en clase desempeñar papeles y efectuar actividades sociales.

Esta distinción entre "tarea” y "acción", al igual que la definición abstracta de "tarea" anteriormente presentada, permite elaborar otro modelo eficaz para analizar las metodologías sucesivas y a la vez diversificar los dispositivos de aprendizaje/enseñanza:

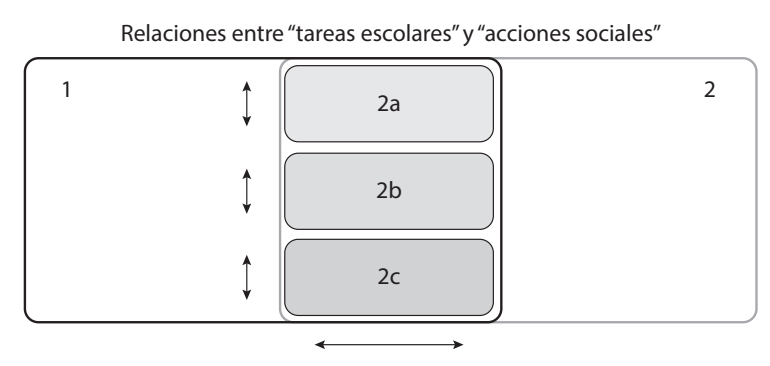

1. La sociedad como área de acciones con finalidad social 
2. La clase como área de tareas con finalidad didáctica (aprendizaje/enseñanza)

2a. La clase como micro-sociedad auténtica

2b. La simulación

2c. La clase como lugar de concepción y realización de acciones de con finalidad social

Cada metodología, y más allá cada pedagogía, amplía o al contrario limita el área de intersección entre los conjuntos 1 y 2 , y otorga más o menos importancia respectiva a los diferentes tipos (2a, 2b, 2c) de intersección. En la "pedagogía Freinet", por ejemplo, se da a las áreas 2a y 2 c la mayor importancia, mientras que en el enfoque comunicativo se privilegia masivamente el área $2 \mathrm{~b}$, a expensas de las otras dos áreas, las que naturalemente tienen que privilegiar los profesores de lenguas de la enseñanza escolar si quieren cumplir con su responsabilidad paralela de educadores. A este modelo como al anterior (el de las diferentes orientaciones de las tareas) se debe aplicar el paradigma de la adecuación: cada profesor debe ser capaz de modular la importancia respectiva de esas diferentes intersecciones en función de las variaciones de su entorno y de sus dispositivos de enseñanza.

Si cruzamos este modelo con el anterior, notamos que en la zona 2 se sitúan las orientaciones lengua, proceso y procedimiento, la orientación producto en la zona 2c, y la comunicación simulada en la zona 2b. En cuanto a la orientación proyecto, su gran flexibilidad le permite cubrir las tres áreas 2a, b, c variando su importancia respectiva, y ésta es la razón por la que la conocida "pedagogía del proyecto" viene a ser la orientación privilegiada naturalmente en "la perspectiva accional" esbozada por los autores del Marco europeo común. Al contrario de lo que pasa en el enfoque comunicativo, en la que se privilegia la comunicación simulada, en esta "perspectiva accional", tal como lo exige por otra parte el paradig- ma de la adecuación, cada uno de los cuatro tipos de comunicación - que podríamos por ejemplo llamar "didáctica" en la zona 2, "escolar" en la zona 2a, "simulada" en la zona $2 \mathrm{~b}$ y "social" en la zona $2 c-$ tienen su interés y su validez.

\section{"Perspectiva accional" y"perspectiva co-accional"}

La expresión "perpectiva accional" utilizada por los autores del Marco europeo común para llamar la nueva orientación didáctica no es del todo satisfactoria, porque en realidad cada metodología constituida (la metodología tradicional gramática-traducción hasta finales del siglo XIX, la metodología directa de principios del siglo XX, la metodología audio-oral norteamericana de los años cincuenta, la metodología audiovisual francesa de los años sesenta, el enfoque comunicativo) tuvo su propia "perpectiva accional" que la diferencia de las otras, por lo menos si damos a esta expresión - como lo hice más arriba con el concepto de "tarea" - su sentido más abstracto, el de "representación de la relación entre el aprendizaje de la lengua extranjera (las tareas) y su uso social (las acciones)". Cada una de estas metodologías históricas elaboró su perspectiva accional en función de un "objectivo social de referencia" diferente, objetivo que corresponde precisamente al conjunto de las acciones sociales que se quería que los alumnos fueran capaces de realizar en lengua extranjera al final de su formación. Si consideramos toda la evolución metodológica de los últimos cien años en Europa, nos damos cuenta de que la especificidad de la reciente perspectiva accional del Marco europeo común es la de ser una perspectiva que propongo llamar "co-accional" porque pone énfasis en la dimensión colectiva de las acciones y en la finalidad social de las mismas. Mientras que en la época de los "Niveles umbrales", a principios de los setenta, este objetivo era la gestión correcta de los encuentros puntuales con extranjeros (y por lo tanto el intercambio más rápido y eficaz de informaciones: cf. el pair work y 
el information gap...), los progresos de la integración económica y política en los años dos mil exigen un objetivo más ambicioso, i.e. la capacidad, para cada ciudadano europeo, de vivir y trabajar con extranjeros. Por eso, en la didáctica europea que está emergiendo en estos últimos años, claramente hemos salido del enfoque comunicativo y del enfoque por tareas que habían sido elaborado en su marco, pasando en particular del concepto comunicativo de interacción (que es un hablar con otro actuando sobre él) al concepto de co-acción (que es un actuar con los otros). ${ }^{7}$

No se trata aquí, por parte mía, de sostener que la perspectiva co-accional debería reemplazar de ahí en adelante el enfoque comunicativo en los contenidos de formación docente, los manuales y las clases. En coherencia con el paradigma de la adecuación, yo diré que lo único que hay que abandonar es la concepción tradicional del progreso por sustitución, cambiándola por la concepción moderna del progreso por enriquecimiento: la perpectiva co-accional esbozada por el Consejo de Europa a finales de los 90 ensancha el abanico de las herramientas didácticas (coherencias, principios, enfoques, procedimientos, técnicas, métodos, dispositivos, etc.), pero sólo el profesor en su clase, como profesional responsable, puede decidir cuáles de ellas (incluidas las más "tradicionales") serán las más eficaces por ser las más adecuadas a sus alumnos y a su entorno global de enseñanza/aprendizaje.

\section{REFERENCIAS}

Nunan, D. (1989). Designing Tasks for the Communicative Classroom. Cambridge: University Press.

7 En cuanto a esta evolución histórica y a las consecuencias didácticas previsibles de esta nueva perspectiva coaccional, remito a : Christian Puren, "Perspectives actionnelles et perspectives culturelles en didactique des langues-cultures : vers une perspective co-actionnelle co-culturelle", Les Langues modernes, ${ }^{\circ} 3 / 1992$, jul.sept., pp. 55-71. Paris, APLV. Está prevista en 2004 la publicación de la versión española de este artículo. 